\title{
Diagnostic and therapeutic utility of transrectal ultrasound in urological office prostatic abscess management: A short report from a single urologic center
}

\author{
Andrea Fabiani ${ }^{1}$, Alessandra Filosa ${ }^{2}$, Valentina Maurelli ${ }^{1}$, Fabrizio Fioretti ${ }^{1}$, Lucilla Servi ${ }^{1}$, \\ Mara Piergallina ${ }^{1}$, Giovanni Ciccotti ${ }^{1}$, Matteo Talle' ${ }^{1}$, Gabriele Mammana ${ }^{1}$ \\ ${ }^{1}$ Surgery Dpt, Section of Urology, ASUR Marche Area Vasta 3, Macerata Hospital, Macerata, Italy; \\ 2 Section of Pathological Anatomy, Department of Clinical Pathology, Area Vasta 3, ASUR Marche, Macerata Hospital, Macerata, Italy
}

\section{Summary Objectives: Prostatic abscess (PA) is an infrequent condition in the modern antibiotic era. The everyday use of transrectal ultra-} sound (TRUS) during diagnostic work-up and the widespread recurrence to prostatic biopsies may lead to an increase of PA diagnosis. In this short report we analyze the patients characteristics and the management of seven recent cases of PA diagnosed in our institution.

Materials and Methods: The records of 7 patients admitted to our Center for LUTS associated to septic fever or acute urinary retention, was prospectively collected. Suspect of PA was done on digital rectal examination (DRE) and confirmed by TRUS performed after urinary system ultrasound (UUS) evaluation. Patients were admitted to hospital only in case of septic signs. A sovrapubic (SPC) or urethral catheter (UC) was placed depending on symptoms. A TRUS-guided aspiration of PA was performed with patient in lithotomic position, using a 18 gauge two-part needle, side/end fire needle access. Patient was discharged with antibiotic therapy and followed up until complete resolution of the PA and symptoms.

Results: Mean age was 62 years (range 24-82). Two patients were diabetics and one was affected by the immunodeficiency acquired syndrome (HIV). In one case, PA was detected after a persistent fever post TRUS guided prostate biopsy. Average prostate volume was $69 \mathrm{ml}$ (range 19-118 ml). DRE was able to diagnose PA only in 2 cases (29\%), UUS evaluation in 1 case (14\%). All cases were confirmed by TRUS as hypo-anechoic areas with or without internal echoes in all patients. Mean PA dimension was $3.64 \mathrm{~cm}$ (range 1.5-8). SPC was placed in 3 cases (43\%), UC in 3 patients (43\%). Only 1 patient refused catheterization. Side fire needle aspiration was performed in all cases and in combination with end fire access in case of particular location of abscess cavities. Second look was needed in 2 cases (29\%). Antibiotics were administered in all cases. The aspirated pus showed a positive culture for Escherichia coli (43\%), Klebsiella pneumoniae (29\%), Pseudomonas aeruginosa (14\%) and Enterococcus faecalis (14\%). PA resolution time mean was 9 days (range 3-24).

Conclusions: TRUS evaluation in case of persistent LUTS associated with fever or acute urinary retention is determinant in the diagnosis of PA. Office or institutional management with TRUS needle aspiration is a good option in these cases.

KEY WORDS: Prostatic abscess; TRUS; End fire probe; Side fire access LUTS; Uroseptic fever.

Submitted 3 October 2014; Accepted 31 October 2014

\section{INTRODUCTION}

Prostatic abscess (PA) is an infrequent condition in the modern antibiotic era. Nevertheless, the wide use of antibiotics in patients with lower urinary tract symptoms (LUTS) could be responsible of the growing PA incidence in the last years (1). The diagnosis may be difficult because at onset of the symptoms PA may mimic several other diseases of the lower urinary tract. Simple interpretation of the clinical symptoms and digital rectal examination (DRE) could be not able to make a diagnosis. The routine use of transrectal ultrasound (TRUS) evaluation in each case of LUTS associated to fever or predisposing factors for the development of PA may aid the clinician in the daily practice (2). In this short report we present data about seven patients diagnosed with prostatic abscess, discussing clinical findings, diagnostic criticisms and treatment results obtained by TRUS guided needle aspiration.

\section{MATERIAL AND METHODS}

We prospectively collected the clinical and instrumental data of a short series of 7 patients admitted from Emergency Department to our Section of Urology in Macerata Hospital for LUTS associated to septic fever and/or acute urinary retention in a 12 months period. At the urologic evaluation, the patients underwent to DRE and systematically to TRUS after an urinary system ultrasound (UUS) study. In case of confirmed suspect of PA, a sovrapubic (SPC) or urethral catheter (UC) was placed depending on symptoms. A TRUS-guided needle aspiration of PA was performed with patient in lithotomic position, using a 18 gauge two-part needle, with a probe $\mathrm{B}-\mathrm{K}$ Type 8818 (BK Medical, Denmark), side/end fire needle access, without local anesthesia. All procedures were performed in an ambulatory setting. Patients were admitted to hospital only in case of septic signs. The patients were discharged with antibiotic therapy and followed up with TRUS until complete resolution of the PA and symptoms.

\section{RESULTS}

Diagnostic and management data are presented in Tables 1 and 2. The figures show particular aspects of PA diag- 
Table 1. Diagnostic findings of cases series.

\begin{tabular}{|c|c|c|c|c|c|c|c|}
\hline Patient & Age & Co-morbidities & Clinical presentation & DRE diagnostic + & UUS diagnostic * & Abscess location & Pathogen \\
\hline 1 & 61 & $\mathrm{BPH}$ & $\begin{array}{l}\text { Fever post TRUS biopsies } \\
\text { (24 cores)* }\end{array}$ & + & & TZ & K. Pneumoniae \\
\hline 2 & 70 & $\mathrm{BPH}$ & LUTS and fever & & & $\mathrm{PZ}$ & Pseudomonas \\
\hline 3 & 68 & DMNID & Fever & & * & TZ PZ & K. Pneumoniae \\
\hline 4 & 24 & Infertility & $\begin{array}{l}\text { AUR and fever after } \\
\text { recurrent hematospermia }\end{array}$ & & & TZ & E.Coli \\
\hline 5 & 82 & $\mathrm{BPH}$ & Fever* & & & TZ & E.Coli \\
\hline 6 & 60 & DMNID & Fever and AUR & & & TZ & Enterococcus faecalis \\
\hline 7 & 69 & HIV & Fever* & + & & TZ PZ & E. Coli \\
\hline
\end{tabular}

Table 2. Technical management of cases.

\begin{tabular}{|c|c|c|c|c|c|c|c|}
\hline Patient & Age & $\begin{array}{l}\text { Prostate volume }(\mathrm{ml}) / \\
\text { abscess diameter }(\mathrm{cm})\end{array}$ & $\begin{array}{l}\text { Soprapubic catheter } \\
\text { positioned * }\end{array}$ & $\begin{array}{l}\text { Urethral catheter } \\
\text { positioned }^{\circ}\end{array}$ & $\begin{array}{l}\text { Side fire (SF)/ } \\
\text { Side fire + end fire } \\
\text { (SFEF) accesss }\end{array}$ & $\begin{array}{c}\text { Second look } \\
\text { needle aspiration + }\end{array}$ & $\begin{array}{l}\text { Time resolution } \\
\text { (days) }\end{array}$ \\
\hline 1 & 61 & $75 \mathrm{ml} / 3,9 \mathrm{~cm}$ & $*$ & & SF & & 7 \\
\hline 2 & 70 & $71 \mathrm{ml} / 3,1 \mathrm{~cm}$ & & & SF & & 5 \\
\hline 3 & 68 & $69 \mathrm{ml} / 4,9 \mathrm{~cm}$ & * & & SFEF & + & 9 \\
\hline 4 & 24 & $19 \mathrm{ml} / 2,1 \mathrm{~cm}$ & & $\circ$ & SF & & 9 \\
\hline 5 & 82 & $118 \mathrm{ml} / 8 \mathrm{~cm}$ & & $\circ$ & SFEF & & 6 \\
\hline 6 & 60 & $68 \mathrm{ml} / 1,5 \mathrm{~cm}$ & & $\circ$ & SFEF & & 3 \\
\hline 7 & 69 & $61 \mathrm{ml} / 2,3$ & * & & SFEF & + & 24 \\
\hline
\end{tabular}

nosis and treatment in same patients. Mean age was 62 years (range 24-82). Two patients were diabetics and one was affected by the immunodeficiency acquired syndrome (HIV). In one case, PA was detected after a persistent fever post TRUS guided prostate biopsy ( 24 biopsy cores). Average prostate volume was $69 \mathrm{ml}$ (range 19$118 \mathrm{ml}$ ). DRE was able to diagnose PA only in 2 cases (29\%), UUS evaluation in 1 case (14\%). PA was confirmed by TRUS (both bi-plane and end-fire probe) as a hypo-anechoic areas with or without internal echoes in all patients. PA was located in transitional zone in 4 cases, peripheral zone was involved in 1 case. Combined localization was observed in 2 cases. PA dimension mean was $3.68 \mathrm{~cm}$ (range 1.5-8). SPC was placed in 3 case (43\%), UC in 3 patients (43\%). Only 1 patient refused catheterization. Side fire needle aspiration was performed in all cases. Combination with end fire needle access was performed in four cases (57\%) due to the localization of abscess cavities. Second look was needed in 2 cases (29\%). Intravenous parenteral antibiotics (Imipenem plus Teycoplanine) was administered only in case of hospitalization (43\%). In cases managed at home, a combination of 3-rd generation Cephalosporine and Aminoglycoside was administered. The aspirated pus showed a positive culture in all patients (Escherichia coli $\mathrm{n}=3$, Klebsiella pneumoniae $\mathrm{n}=2$, Pseudomonas $\mathrm{n}=1$ and Enterococcus faecalis $\mathrm{n}=1$ ). Mean PA resolution time was 9 days (range 3-24). No patients died for this condition.

\section{Discussion}

PA is a potential life threatening condition that, when not adequately treated, may progress to sepsis and death. Thus, an accurate diagnostic and an efficient treatment are both required. In available scientific literature data about PA consisted in cases reports and there is no standardization of the diagnostic and therapeutic routine. In review articles, we found several reports discussing clinical presentation, diagnostic work up and therapeutic approach to PA. The summary of these individual experiences permitted to delineate some lines of action (3-4). First of all, we think that PA incidence will be increasing in the next years. In the modern antibiotic era incidence is estimated between $0.5 \%$ to $2.5 \%$ of diseases accompanying prostatic symptoms. We should expect a shift of the epidemiological profile of PA due to a widespread routine use of broad-spectrum antibiotics to patients with LUTS, without the investigation required (1), and to an increase in population longevity that lead to a further need to manage chronic illness, such as diabetes mellitus or chronic renal failure, promoting the infectious risk. Also others diseases altering the immune system could be responsible, e.g. immunodeficiency 
Figure 1a. Bladder ultrasound:

68 years old, presented at our attention for persistent fever and prostatic abscess (*).

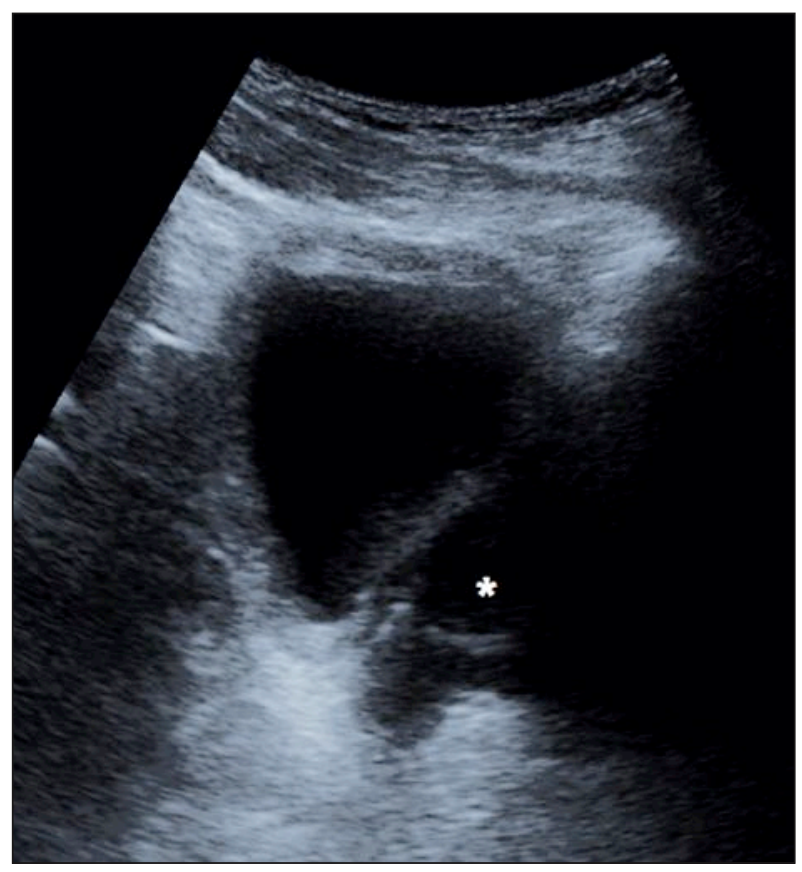

Figure 1b. Transrectal ultrasound (end-fire probe) view of the figure 1 a confirm a multisided prostatic abscess (*).

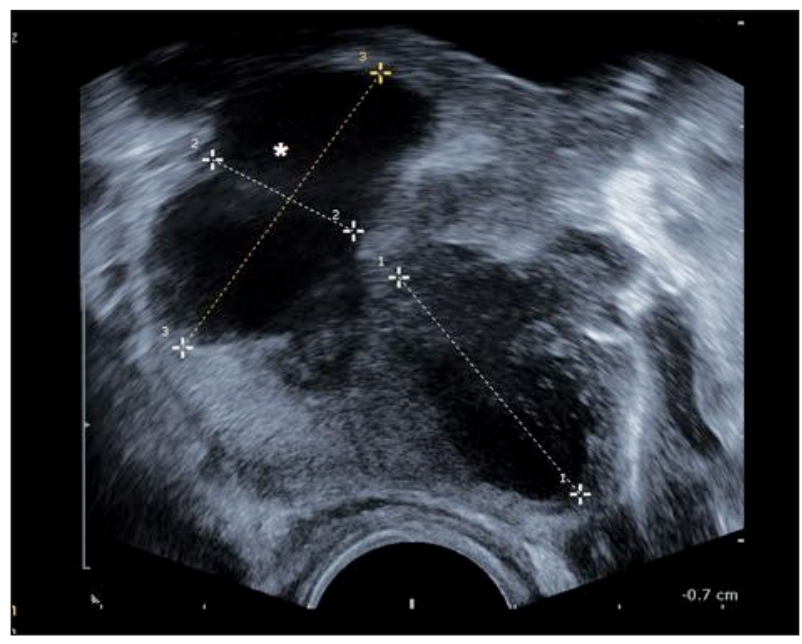

Figure 2a-b.

TRUS evaluation (endfire probe) at follow up of patient in figures 1a-b, axial (A) and longitudinal (B).



acquired syndrome (5). Then, if we recognize that the abscess is a result of the retrograde flow of contaminated urine during micturition into prostatic ducts that promotes the formation of microabscesses that coalesce and form prostatic abscesses (6-7), the rate of this disease is certainly more important than what reported in the scientific literature. Differential diagnosis between acute bacterial prostatitis and PA is difficult if based only on clinical symptoms and digital rectal examination (DRE) (2) or lower abdominal ultrasound evaluation. The routine use of TRUS evaluation in each case of LUTS associated to fever or predisposing factors for the development of PA may aid the clinician in the daily practice (3). At DRE prostatic abscess could be appreciate as a painful fluctuating area (4). In our short report this findings was observed in 2 cases. Similarly, prostate lower abdominal ultrasound evaluation was able to pose the PA suspect only in 1 case in which the exam revealed an hypoechoic irregular area within prostate parenchyma suggesting the diagnosis confirmed by TRUS (Figures $1 \mathrm{a}, 1 \mathrm{~b}$ ). In the other cases, abdominal ultrasound was unremarkable. In our experience TRUS showed to be an excellent tool in making the diagnosis. The most common finding is the presence of one or more hypo-anechoic areas, of several sizes, containing thick liquid and located in the transition zone and/or in central zone of the prostate, permeated by hyperechogenic areas and anatomical gland distortion. In our series these findings were variably observed in $100 \%$ of the cases. Although other conditions could have a similar TRUS appearance, as neoplastic process, cystic lesions and granulomas (8-10), the TRUS appearance of PA is quite characteristic and can be differentiated on the basis of determined criteria (11). As reported in the literature, TRUS should also be considered the diagnostic study of choice to assist the treatment and follow-up of patients with prostatic abscess (12). Figures $2 \mathrm{a}-2 \mathrm{~b}$ show the results of TRUS guided needle aspiration at follow up of one patient. As observed in our cases, clinicians need to suspect PA in case of fever with LUTS especially in patients presenting with fever and persistent LUTS despite antibiotics use, for diabetics or immune-deficient men with protracted symptoms, for those with LUTS and fever progressing to urinary retention and after the performance of prostatic biopsy. In all scientific reports, diabetes mellitus and HIV infection are invariably referred as risk factors for the development of PA. In our series, a peculiarity was represented by case 4 (Figure 3 ) that was a young patient ( 24 years old) who developed AUR due to infectious enlargement of a midline cyst and was symptomatic for recurrent hematospermia, as revealed by clinical history taken at the moment of emergency evaluation. These results highlight the importance to investigate with TRUS younger patients with genito-urinary symptoms (13). In reference to prostate biopsy as risk factor of PA, it should be underlined that data from European Randomized Study of Screening for Prostate Cancer (Rotterdam section) (ERSPC) revealed growing evidence of increasing hospitalizations for serious infectious complications within 2 week of prostate biopsy (14). Specifically, the Authors found a $10 \%$ increase in the frequency of hospital admissions and most of these were for infectious complications 
Figure 3.

A midline prostatic utricle cyst after TRUS guided needle aspiration in young men with acute urinary retention.

Wall cyst presents small calcification.

The surrounding right seminal duct is shown in figure 5.



probably related to rising antimicrobial resistance. It is well known that men with prostatic enlargement and diabetes or major co-morbidities had an increased risk of febrile complications after prostate biopsy (15). These results highlight the importance of judicious patient selection for PSA screening because these men may be less likely to benefit from early prostate cancer detection and also have a greater risk of complications from the diagnostic work-up. However, we considered that in case of patient number 1 of our series, neither prostate volume $(57 \mathrm{ml})$ neither co-morbidities predicted PA onset, due to a multi resistant Klebsiella pneumoniae. In our local experience with TRUS guided biopsies, on 1382 procedures performed in the last 6 years, we found only thissingle case of PA $(0.07 \%)$ and 10 cases of hospitalizations for septic fever $(0.72 \%)$ with 1 admission to intensive care unit. No deaths from this complication was observed (unpublished data). We can confirm that the frequency of hospital admission for septic fever after prostate biopsy is low $(<1 \%)$ (15). The problem is represented by the high level of antimicrobial resistance. We must take into consideration the use of a combination of 3-rd generation Cephalosporine and Aminoglycoside or Imipenem and Teicoplanine as better antibiotic therapy than traditional fluorquinolones. TRUS-guided needle aspiration is the method of choice for treatment of PA (16). The treatment options included also surgical intervention such as transurethral prostate incision (TUIP), transurethral de-roofing (TURP) or transperineal tube placement. TURP is a more invasive approach and it is associated with several risks such as hemorrhage, retrograde ejaculation and sepsis. An alternative to simple aspiration could be the continuous drainage with a tube placed under TRUS guidance either by transperineal or transrectal route. However, this approach is fraught with a serious risk of developing a prostate-rectal fistula formation and prolonged hospitalization (17-18). Tiwari et al. (19) reported 24 patients treated with transurethral de-roofing in 17 cases, transperineal needle aspiration TRUS guided in 3 cases. The remnants patients was managed conservatively. Invasive treatment was applied in case of dimension of cavities abscess $>1 \mathrm{~cm}$ or multi-loculated. Vias et al. (3) published one of the largest series of PA managed with TRUS-guided needle aspiration. Of 48 patients, they report a $100 \%$ of diagnostic accuracy by TRUS and a success treatment rate of $85.42 \%$, avoiding the risk of potential disadvantages of TUR. The diameter "cut off" of cavities considered eligible for aspiration was $2 \mathrm{~cm}$. In our short report, we confirm the high diagnostic accuracy (100\%) of TRUS with a similar success rate. Applying the dimensional cut-off proposed by Vias, only two patient needed a second look aspiration due to the persistence of cavities within the prostate. No TUR de-roofing was performed. In all cases, our TRUS follow up revealed a complete resolution of PA. Technically, our TRUS guided needle aspiration consisted in the use of a biplane probe (6-12Mhz, Type 8818, BK Medical, Denmark) with a side fire needle access in all patients (Figure 4). When PA was multifocal (four patients), especially in two cases in which cavities was located in the anterior zone of the prostate, we resorted to an end fire access (Figure 5) in view of the potential increased capacity of this configuration, showed during prostate biopsies, to sample this anatomical area of the gland (20). We approached all cases with catheterization. Suprapubic catheter was placed in three case. In one case, patient refused catheterization both urethral and suprapubic. Also in this case the evolution of treatment was positive without need of ancillary procedures.

\section{Figure 4.}

TRUS guided aspiration with a biplane probe, side fire needle access.

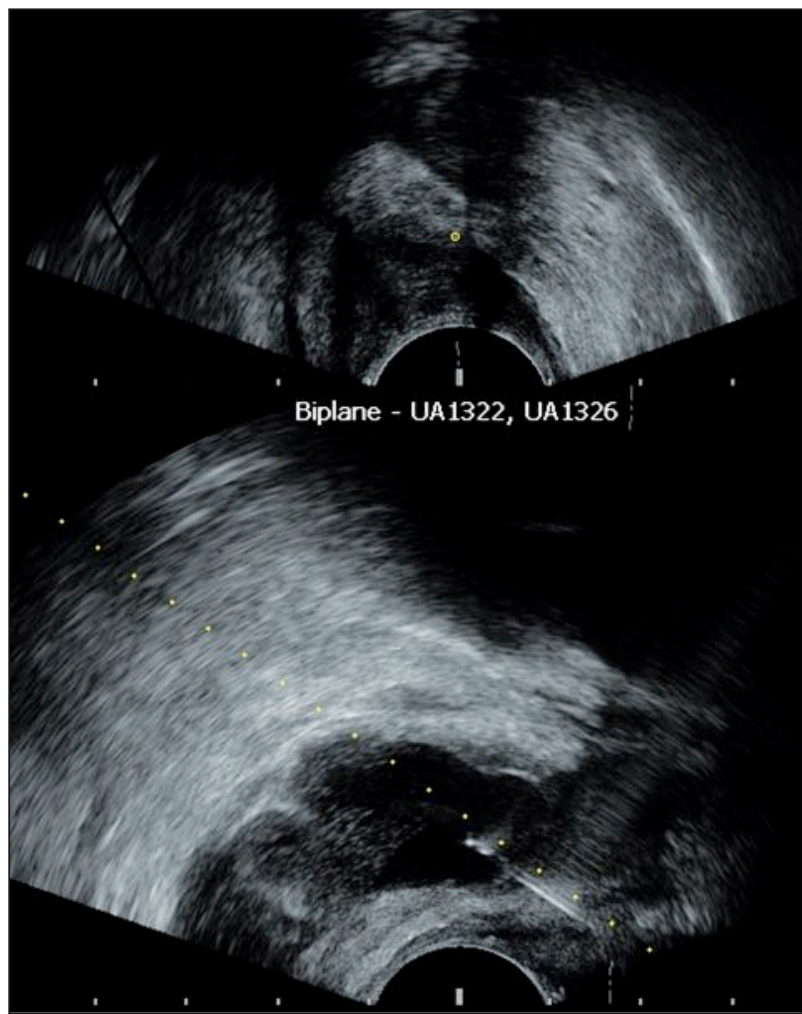




\section{Figure 5.}

Longitudinal view of patient in figure 3: TRUS guided aspiration with an endfire probe, endfire needle access.

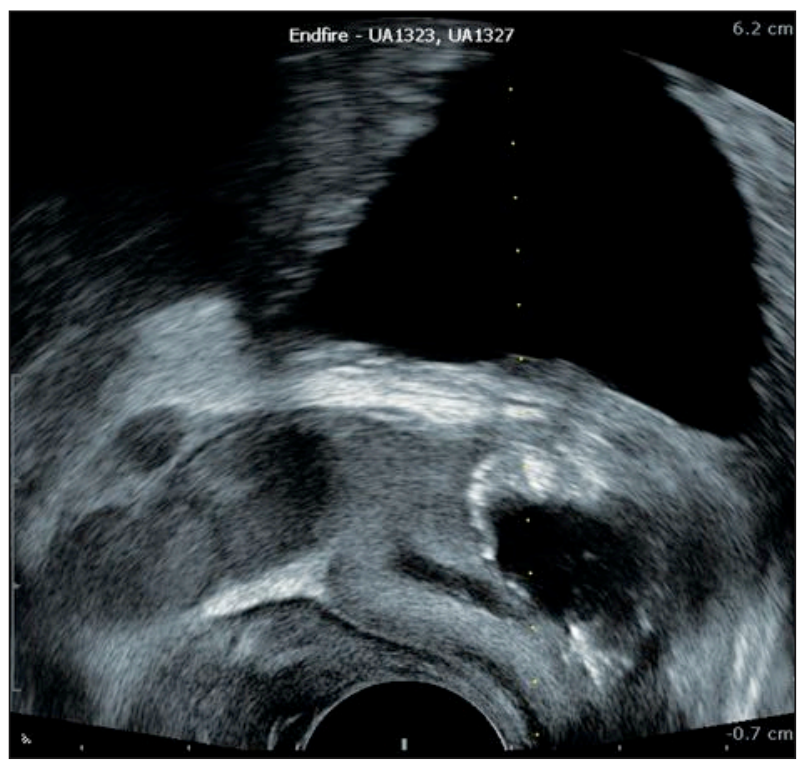

\section{Conclusions}

The diagnosis of prostatic abscess should be warranted for patients presenting with fever and persistent LUTS despite antibiotics use, for diabetics or immune-deficient patients with protracted symptoms and for those with LUTS and fever progressing to urinary retention and after the performance of prostatic biopsy. TRUS evaluation is determinant and mandatory for the diagnosis.. In our experience, office or institutional management with TRUS needle aspiration is a good option in all cases.

\section{REFERENCES}

1. Granados EA, Riley G, Salvador J, Vicente J. Prostatic abscess: Diagnosis and treatment. J Urol. 1992; 148:80-2

2. Oliveira P, Andrade JA, Porto HC, et al Diagnosis and treatment of prostatic abscess Int Braz J Urol. 2003; 29:30-4.

3. Vias BJ, Ganpule SA, Ganpule AP, et al. Transrectal ultrasoundguided aspiration in the management of prostatic abscess: A singlecenter experience Indian J Radiol Imaging. 2013; 23:253-257.

4. Granados EA, Caffaratti J, Farina L, Hocsman H: Prostatic abscess drainage: Clinical-sonography correlation. Urol Int. 1992; 48:358-61.

5. Trauzzi SJ, Kay CJ, Kaufman DG, Lowe FC. Management of prostatic abscess in patients with human immunodeficience syndrome. Urology. 1994; 43:629-33.

6. Meares EM, Jr. Prostatic abscess. J Urol. 1996; 129:1281-2.

7. Porfyris O, Kalomoiris P. Prostatic abscess: Case report and review of the literature Arch Ital Urol Androl. 2013; 85,3:154-6.

8. Galosi AB, Parri G, Lacetera V. et al. Management of large prostatic abscess associated with urethral stenosis and penile cancer recurrence. Arch Ital Urol Androl. 2010; 82:181-5.

9. Galosi AB, Montironi R, Fabiani A, et al. Cystic Lesions of the Prostate Gland: An Ultrasound Classification With Pathological Correlation J Urol. 2009; 181:647-657.
10. Hamper UM, Epstein JI, Sheth S, et al. Cystic lesions of the prostate gland: A sonographic-pathologic correlation. J Ultrasound Med. 1990; 9:395-402.

11. Barozzi L, Pavlica P., Menchi I., et al. Prostatic abscess: Diagnosis and treatment. AJR Am J Roentgenol 1998;170:753-7

12. Lee F Jr, Lee F, Solomon MH, et al. Sonographic demonstration of prostatic abscess. J Ultrasound Med. 1986; 5:101-2.

13. Akhter W., Khan F., Chinegwundoh F. Should every patient with hematospermia be investigated? A critical review Cent European J Urol. 2013; 66:79-82

14. Loeb S, van den Heuvel S, Zhu X, et al. Infectious Complications and Hospital Admissions After Prostate Biopsy in a European Randomized Trial Eur Urol 2012; 6 1:1110-14.

15. Loeb S, Carter HB, Berndt SI, et al. Complications after prostate biopsy: data from SEER-Medicare. J Urol. 2011; 186:1830-4.

16. Lim JW, Ko YT, Lee DH, et al. Treatment of prostatic abscess: Value of transrectal ultrasonographycally guided needle aspiration. J Utrasound Med. 2000; 19:609-17.

17. Aravantinos E, Kalogeras N, Zygoulakis N, et al. Ultrasoundguided trasrectal placement of drainage tune as therapeutic management of patients with prostatic abscess. Journal of endourology. 2008; 22:1751-4.

18. Arrabal-Polo MA, Jimenez-Pacheco A, Arrabal-Martin M. Percutaneous drainage of prostatic abscess: Case report and literature review. Urol Int. 2012; 88:118-20.

19. Tiwari P, Pal KD, Tripathi A. et al. Prostatic abscess: diagnosis and management in the modern antibiotic era. Saudi J Kidney Dis Transpl. 2011; 22:298-301.

20. Galosi AB, Tiroli M, Cantoro D, et al. Biopsy of the anterior prostate gland: technique with end-fire transrectal ultrasound. Arch Ital Urol Androl. 2010; 82:248-52.

\section{Correspondence}

Andrea Fabiani, MD (Corresponding Author)

andreadoc1@libero.it

Valentina Maurelli, MD

valentinamaurelli@hotmail.it

Fabrizio Fioretti, $M D, P h D$

fa.fioretti@libero.it

Lucilla Servi, MD

lucilla.servi@sanita.marche.it

Mara Piergallina, MD

mara.piergallina@tiscali.it

Giovanni Ciccotti, MD

giovanni.ciccotti@sanita.marche.it

Matteo Talle', MD

matteo.talle@gmail.com

Gabriele Mammana, MD

gabriele.mammana@sanita.marche.it

Surgery Dpt, Head of Section of Urology

ASUR Marche Area Vasta 3, Macerata Hospital, Macerata, Italy

Alessandra Filosa, MD, PhD

alessandrafilosa@yahoo.it

Section of Pathological Anatomy, Department of Clinical Pathology,

Area Vasta 3, ASUR Marche, Macerata Hospital, Macerata, Italy 